\title{
Kinetics for Reduction of Iron Ore Based on the Phase Space Reconstruction
}

\author{
Guo-Feng Fan, ${ }^{1}$ Li-Ling Peng, ${ }^{1}$ Wei-Chiang Hong, ${ }^{2,3}$ and Fan Sun ${ }^{1}$ \\ ${ }^{1}$ College of Mathematics \& Information Science, Pingdingshan University, Pingdingshan, Henan 467000, China \\ ${ }^{2}$ Department of Information Management, Oriental Institute of Technology, No. 58, Section 2, Sichuan Road, \\ Panchiao, Taipei 220, Taiwan \\ ${ }^{3}$ Management School, Hangzhou Dianzi University, Hangzhou, Zhejiang 310018, China
}

Correspondence should be addressed to Guo-Feng Fan; guofengtongzhi@163.com

Received 24 March 2014; Revised 12 June 2014; Accepted 18 June 2014; Published 17 August 2014

Academic Editor: Jiangang Zhang

Copyright (C) 2014 Guo-Feng Fan et al. This is an open access article distributed under the Creative Commons Attribution License, which permits unrestricted use, distribution, and reproduction in any medium, provided the original work is properly cited.

A series of smelting reduction experiments has been carried out with high-phosphorus iron ore of the different bases and heating rates by thermogravimetric analyzer. The derivative thermo gravimetric (DTG) data have been obtained from the experiments. After analyzing its phase space reconstruction, it is found that DTG phase portrait contains with a clear double " $\infty$ " attractor characteristic by one-order delay. The statistical properties of the attractor inside and outside the double " $\infty$ " structures are characterized with interface chemical reaction control and diffusion control stage in dynamic smelting process, respectively; the results are deserved to be a reference value on understanding of the mechanism and optimization and control of the process in smelting reduction of high-phosphorus iron ore.

\section{Introduction}

Chaos theory suggests that the randomness within deterministic system is often caused by the nonlinear dynamic factors instead of random factors from the outside. There are complex nonlinear dynamical systems in practical problems, because of the limit of measuring means; and the unknown information of complex systems and absence of physical quantities contained make it difficult to establish the directly analytical form of the mathematical model. Therefore, the reconstruction of nonlinear dynamical systems uses the time series of a physical quantity which was got by experiments to obtain the internal and inherent characteristics. Such research is an important part of nonlinear analysis [1]. At present, the chaotic time series reconstruction technique has been more widely used in many practical such as the fluid, geology, economic, but few study in the field of steel metallurgy $[2,3]$, especially on smelting reduction of highphosphorus iron ore. There are more than 100 kinds of complex chemical reactions in the smelting reduction process of high-phosphorus iron ore, and it is very difficult to make mechanism research for each reaction. A large number of domestic and foreign literatures mainly focus on idealized mathematical model (such as the reaction core model and the microparticle model) or numerical simulation, but a lot of revised forms still failed to reflect accurately the details of the smelting reduction process [4-11]. In addition, most studies on thermal analysis kinetics made the mathematical model of the response fraction by the weight loss rate or pure substance. After the first derivative the error of mathematical model increases significantly, which results in that the differential type of response fraction equation cannot express the apparent reaction rate (DTG). The evolutionary behavior of the physical quantity (the apparent reaction rate), influenced by complexity from input and dissipation of the energy in the entire process of smelting reduction system, can reflect the details of the smelting to some extent. In view of the limitations of the subject development, however, understanding of the apparent reaction rate is very limited; thus, its change mechanism is not yet fully understood [12]. This article intends to reconstruct phase space dynamic system of the high-phosphorus iron ore smelting reduction system using the apparent reaction rate time series obtained from TGA experiments on Huimin ore smelting reduction with 
different alkalinity ratios and different heating rates. It aims at improving the understanding and mastery of the mechanism of smelting reduction process by extracting and analysis of the implicit information and dynamic characteristics of the phase space. The highlights of this article are as follows: (1) The nonlinear analysis is employed to the DTG time series firstly; (2) the result shows that the reduction system belongs to random and discrete dynamical system; (3) The DTG phase portrait of one-order delay and two-dimension embed has clear graphical structural feature of double " $\infty$ " attractor; (4) the statistical properties with inside and outside " $\infty$ " of the attractor have verified two kinetic cases.

The rest of the paper is organized as follows. The phase space reconstruction method and its analysis and discussion of the experimental data are shown in Section 2. Mathematical model and analysis of dynamic system for analyzing experimental data will be presented in Section 3; in the meanwhile, some comparison between the two methods is also provided. Finally, a brief summary is discussed in Section 4.

\section{Phase Space Reconstruction and Dynamic Characteristic}

2.1. Phase Space Reconstruction Theory. Phase space reconstruction of the dynamic system is a new technology of time series quantitative analysis [13]. The evolution of a physical quantity in system is associated with other physical quantities; so the amount of information hides in the evolutionary process of the physical by time series. The original law could be extracted and restored from time series data of physical quantity.

Let $\left\{x_{1}, x_{2}, x_{3}, \ldots, x_{N}\right\}$ be time series of a physical quantity. The interactional information with the variable in the system is obtained from its evolution process. Therefore the time series is extended to a three-dimensional or higher dimensional phase space so that the information is fully revealed, and this method is so-called phase space reconstruction by time series.

Phase space reconstruction theory proposed by Takens proves that there is a suitable embedding dimension by which a regular trajectory (attractor) would be restored. In other words, it is a diffeomorphism from its trajectory to the original dynamic system in the reconstructed $R^{m}$ space. All of that has established a solid foundation for reconstruction of complex systems [14].

In addition to this, if time series is long enough without the noise, the delay time $t$ could be arbitrarily chosen. However, the actual attractor dimension $D$ of the system in research is unknown [15]; the delay length of time series is limited with noise; so it is the key to choose the appropriate time delay and embedding dimension [16].

The average mutual information method is employed to obtain a delay time. It is a modification of the classical autocorrelation function method from the perspective of information theory and could distinguish effectively folded and unfolded orbit.
TABLE 1: Mineral composition of Huimin ore (\%).

\begin{tabular}{lcccccccc}
\hline TFe & $\mathrm{FeO}$ & $\mathrm{SiO}_{2}$ & $\mathrm{Al}_{2} \mathrm{O}_{3}$ & $\mathrm{MgO}$ & $\mathrm{CaO}$ & $\mathrm{S}$ & $\mathrm{P}$ & $\mathrm{TiO}_{2}$ \\
\hline 41.02 & 1.36 & 17.98 & 5.60 & 0.21 & 0.35 & 0.06 & 0.9 & 0.28 \\
\hline
\end{tabular}

The calculated results of the autocorrelation function are tended to lag, and the emphasis is a linear correlation part. The average mutual information function includes nonlinear components; the information theory is employed to select $t$ with an iterative fashion. Select the value $t$ corresponding to the first minimum as the best time delay; the track reconstructed using the minimum point afterward will fold. Autocorrelation function has similar defect.

The vector sequence was reconstructed by time delay for time series $x_{1}, x_{2} \ldots x_{N}$ as follows (1) [14]:

$$
\chi_{i}(d)=\left(x_{i}, x_{i+t}, \ldots, x_{i+(d-1) t}\right), \quad i=1,2 \ldots N-d t
$$

where $d$ is the embedding dimension, $t$ is time delay, and $\chi_{i}$ is $i$ th reconstructed vector. The $a(i, d)$ is defined as (2)

$$
a(i, d)=\frac{\left\|\chi_{i}(d+1)-\chi_{n(i, d)}(d+1)\right\|}{\left\|\chi_{i}(d+1)-\chi_{n(i, d)}(d+1)\right\|} \quad i=1,2 \ldots N-d t .
$$

The $\|\cdot\|$ maximum norm in the Euclidean space is defined as (3)

$$
\left\|\chi_{k}(m)-\chi_{l}(m)\right\|=\max _{0 \leq j \leq m-l}\left|x_{k+j t}-x_{l+j t}\right| .
$$

The $\chi_{i}(d+1)$ expresses $i$ th reconstructed vector by the embedding dimension $d+1, n(i, d)$ expresses that $\chi_{n(i, d)}(d)$ is false neighbor point of $\chi_{i}(d)$ in the $d$-dimensional reconstructed phase space, and $1 \leq n(i, d) \leq N-d t$.

Here false neighbor is based on the above definition $\|\cdot\|$.

The mean of all $a(i, d)$ is expressed as (4)

$$
E(d)=\frac{1}{N-d t} \sum_{i=1}^{N-d t} a(i, d) .
$$

It can be seen from embedding theory that if the embedding dimension is $d$, the near two points in $d$-dimensional reconstructed phase space should still be closed in the $d+$ 1-dimensional reconstructed phase space. The two points which met this condition are called true neighbor points, or the fake close neighbors. Therefore, if $d \geq D, E(d+1) / E(d)$ should no longer be changed, and its value is one, where $D$ is the minimum embedding dimension.

2.2. Experimental. The TGA experiment equipment is produced by Netzsch, modeling as STA 449 F3. It is protected with nitrogen gas flowing at $50 \mathrm{~mL} / \mathrm{min}$ and argon, acting as purge gas, flowing at a rate of $10 \mathrm{~mL} / \mathrm{min}$.

Raw materials employed in the experiment are taken from the Huimin high-phosphorus iron ore in Yunnan province. The composition of Huimin ore and auxiliary raw materials such as coal and $\mathrm{CaO}$ is shown in Tables 1, 2, and 3. They are in the uniform state after crushing and grinding. As a reducing 


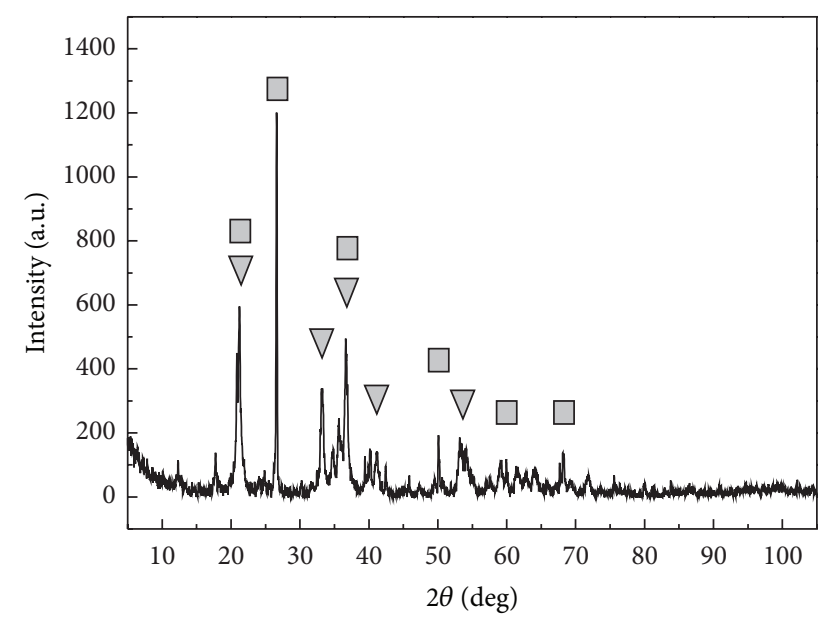

$$
\nabla-\mathrm{FeO}(\mathrm{OH})
$$$$
\square-\mathrm{SiO}_{2}
$$

FIGURE 1: XRD collection of illustrative plates of Huimin iron ore.

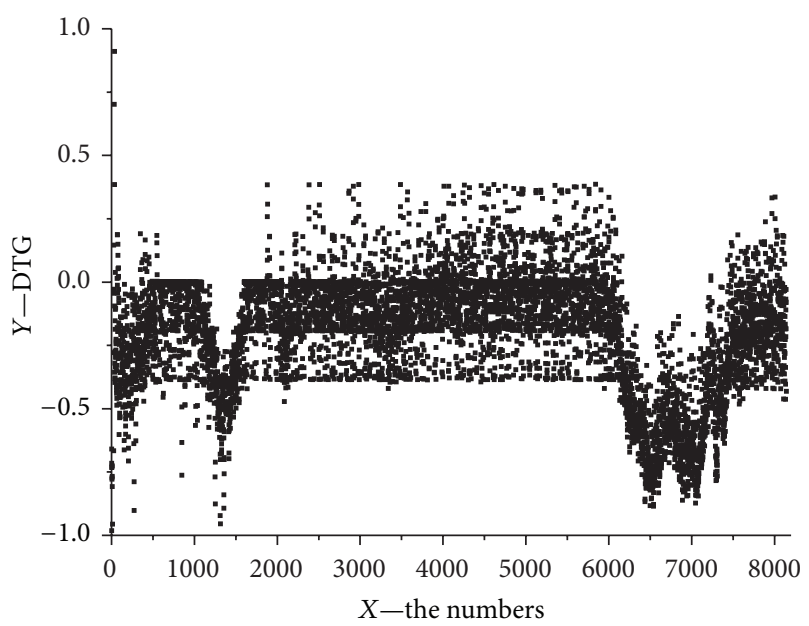

FIgURE 2: The time series of DTG.

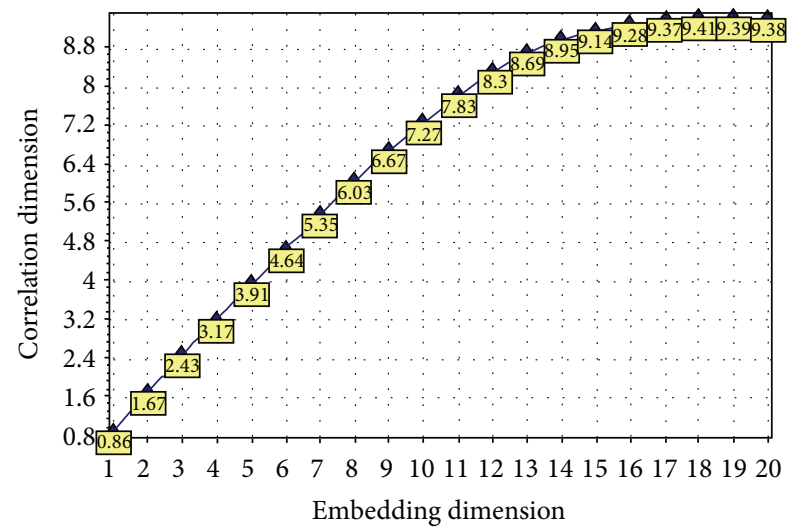

FIgURE 3: Evolution diagram of correlation dimension.

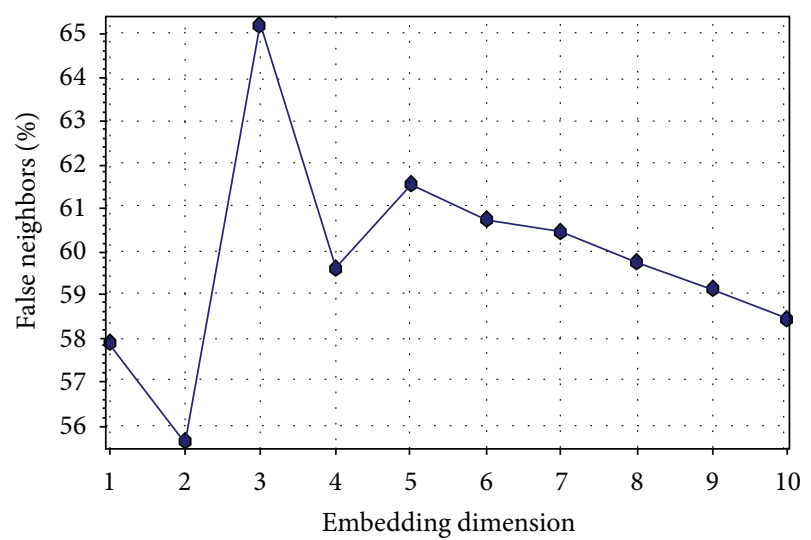

FIGURE 4: Calculated process of optimal embedding dimension.

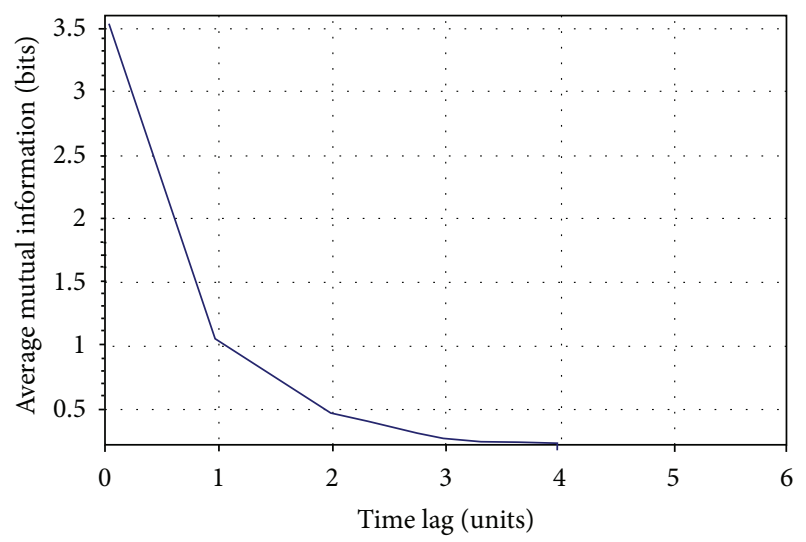

Figure 5: Calculated process of optimal time delay.

TABLE 2: The ingredients of solvent (\%).

\begin{tabular}{cccccc}
\hline $\mathrm{CaO}$ & $\begin{array}{c}\text { Alkali-metal and } \\
\mathrm{MgO}\end{array}$ & $\mathrm{Fe}$ & $\begin{array}{c}\text { Ammonia } \\
\text { precipitate }\end{array}$ & Sulfate & $\begin{array}{c}\text { Loss On } \\
\text { ignition }\end{array}$ \\
\hline 96.3 & 0.5 & 0.015 & 0.2 & 0.1 & 2 \\
\hline
\end{tabular}

agent, the coal in the reaction vessel can maintain a strong reduction atmosphere to make sure that the iron in smelting slag is fully reduced. In addition, the added $\mathrm{CaO}$ can increase the basicity in the slag of reduction process to improve their liquidity and to make iron oxide fully reduced.

As shown in Figure 1, the main phases of Huimin iron ore are $\mathrm{Fe}_{2} \mathrm{O}_{3} \cdot \mathrm{H}_{2} \mathrm{O}$ and $\mathrm{SiO}_{2}$. Huimin limonite ore is a kind of water goethite.

The particle size ranged from 0.001 to $0.1 \mathrm{~mm}$. There are two genetic types for the limonite ore, one is the sedimentary limonite, which was implicit and crystalline aggregate; the other is the limonite aggregate formed by exogenous effect of oxidation and hydrolysis.

2.3. Analysis of Phase Space Reconstruction. Derivative thermogravimetric data of Huimin ore reflect the change of the apparent reaction rate in the smelting reduction process. It is shown in Figure 2. Experiment extent is confirmed with bases 


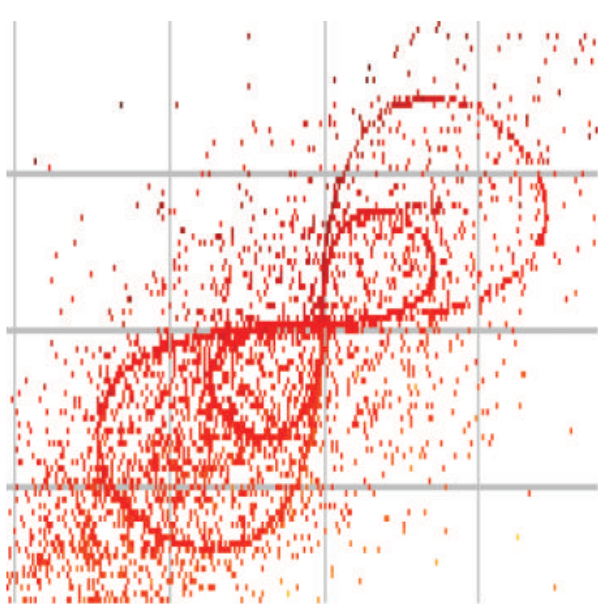

(a)

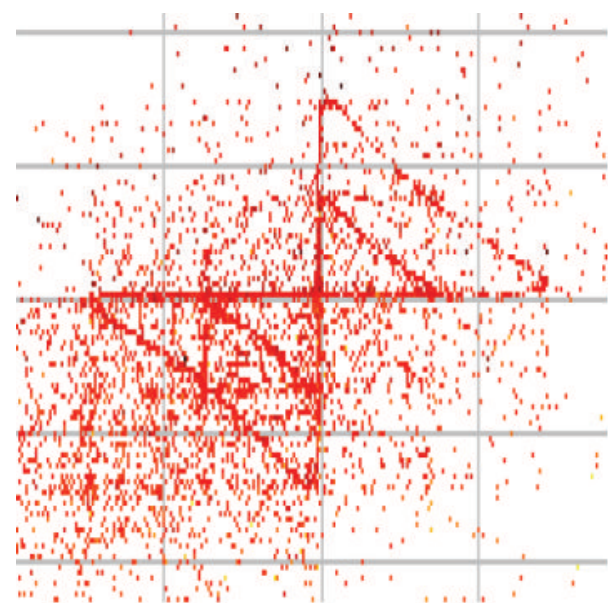

(b)

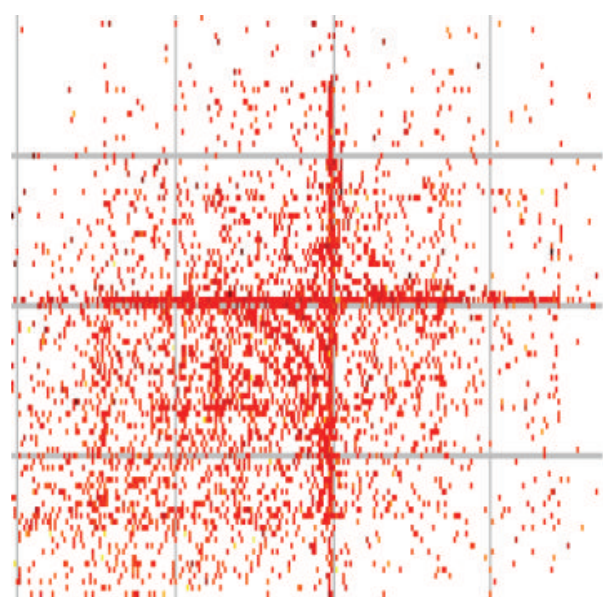

(c)

Figure 6: (a) The phase diagram for $t=1$, (b) the phase diagram for $t=2$, and (c) the phase diagram for $t=3$. (The horizontal axis and vertical axis are, respectively, $x(t)$-DTG and $x(t+1)$-DTG, the colour indicated the dimension).

TABLE 3: The composition of anthracite (\%).

\begin{tabular}{lcccccc}
\hline $\mathrm{C}$ & $\mathrm{CaO}$ & $\mathrm{SiO}_{2}$ & $\mathrm{~S}$ & $\mathrm{P}$ & Ash & Volatile \\
\hline 76.43 & 1.59 & 7.80 & 0.35 & 0.05 & 15.29 & 7.78 \\
\hline
\end{tabular}

TABLE 4: Compositions of each type of materials required.

\begin{tabular}{lccccc}
\hline & \multicolumn{5}{c}{ Bases $(R)$} \\
& 0.8 & 1.1 & 1.4 & 1.7 & 2.0 \\
\hline Mineral content (mg) & 7.48 & 6.85 & 6.82 & 6.53 & 6.37 \\
Coal content (mg) & 1.40 & 1.17 & 1.16 & 1.11 & 1.09 \\
Calcium oxide content (mg) & 1.12 & 1.98 & 2.02 & 2.36 & 2.54 \\
\hline
\end{tabular}

$0.8,1.1,1.4,1.7$, and 2.0 , respectively, and $5,10,15$, and $20 \mathrm{~K} / \mathrm{min}$ in 1.4; the compositions of each type of materials required are shown in Table 4.

Firstly, the features of DTG are analyzed, and then correlation dimension, an important evidence to establish the existence of chaotic continuous systems, is calculated with the reconstruction map of the fractal dimension. For the truly random signals, the correlation dimension graph will look like a 45-degree straight line. It indicates that no matter how the degree of the embedded noise is, it will evenly fill that space. Chaotic (or periodic) signals have a distinct spatial structure, and their correlation dimension will saturate at some point while embedding dimension is increasing.

By calculating the correlation dimension of DTG data to ensure that the correlation dimension will reach saturation status as shown in Figure 3, It is indicated that the nonlinear chaotic characteristics exist in system.

As can be seen from the calculated results in Figures 4 and 5 , the proportion of false is closed to the minimum value when $m=2$ with the embedded dimension increase; then, the proportion begins to increase along with the increasing embedded dimension. Therefore, it is concluded that the embedded dimension $m=2$ is the real nearby point and the optimal embedded dimension should be set as 2 . It can be seen from Figure 6 that the phase diagrams for time delay $t=1$ can reflect the ordered characteristic in the entire reducing process, on the contrary, the cases for $t=2$ or 3 cannot. In addition, it is shown in Figure 5 that the average 


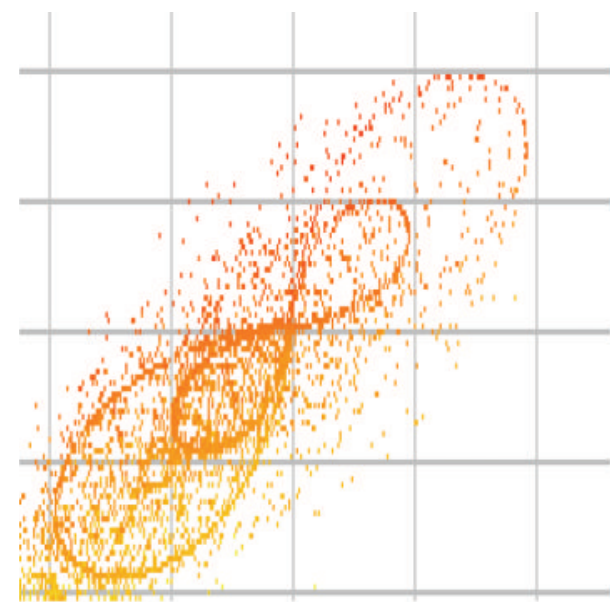

(a)

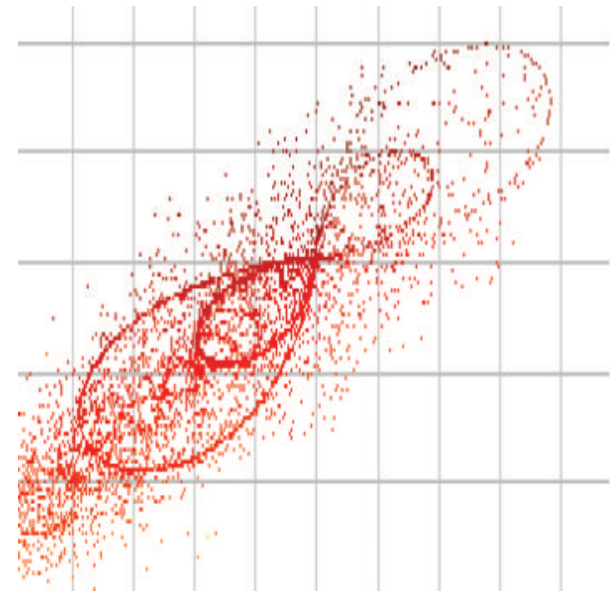

(c)

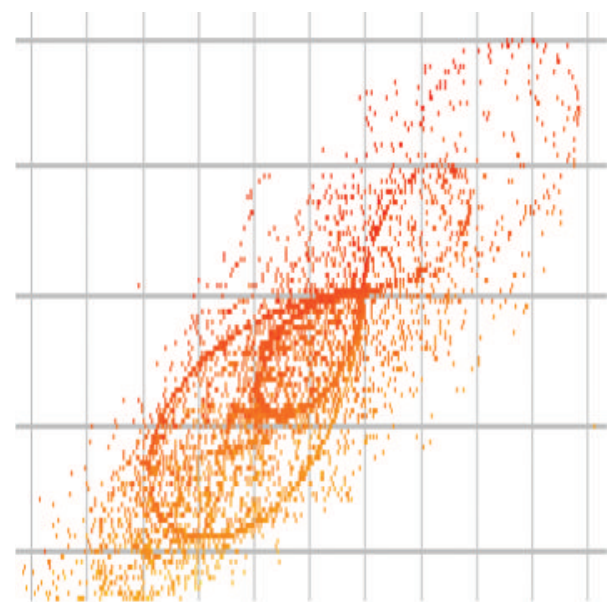

(b)

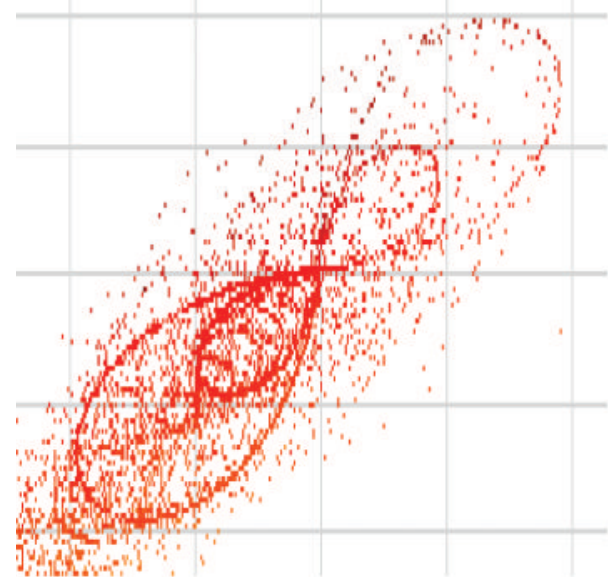

(d)

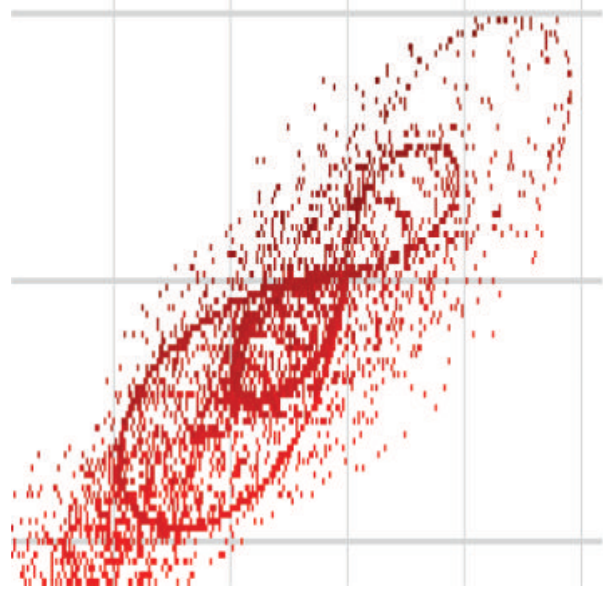

(e)

FIGURE 7: (a) Heating rate of $10 \mathrm{~K} / \mathrm{min}, R=0.8$, (b) heating rate of $10 \mathrm{~K} / \mathrm{min}, R=1.1$, (c) heating rate of $10 \mathrm{~K} / \mathrm{min}, R=1.4$, (d) heating rate of $10 \mathrm{~K} / \mathrm{min}, R=1.7$, and (e) heating rate of $10 \mathrm{~K} / \mathrm{min}, R=2.0$. (The horizontal axis and vertical axis are, respectively, $x(t)$-DTG and $x(t+1)$-DTG, the colour indicated the dimension). 


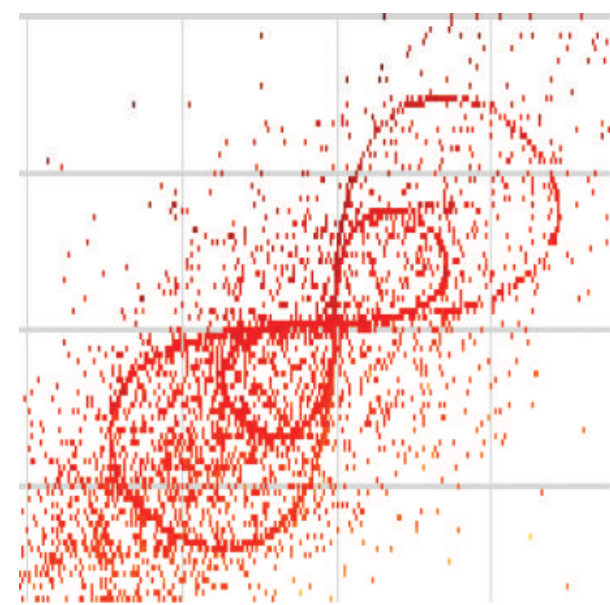

(a)

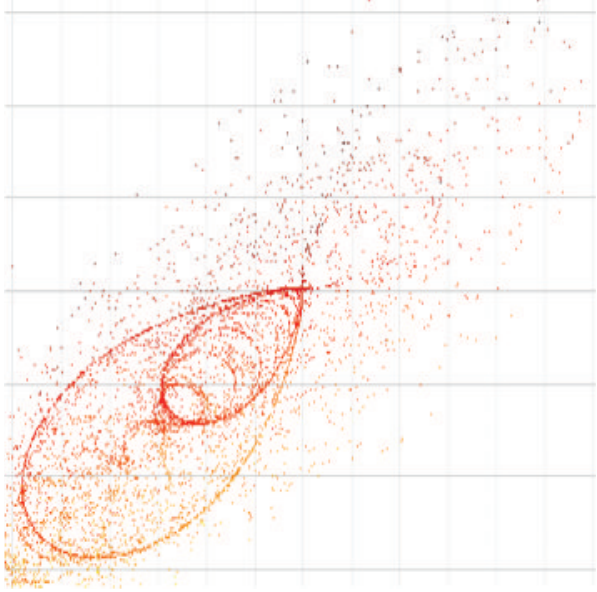

(c)

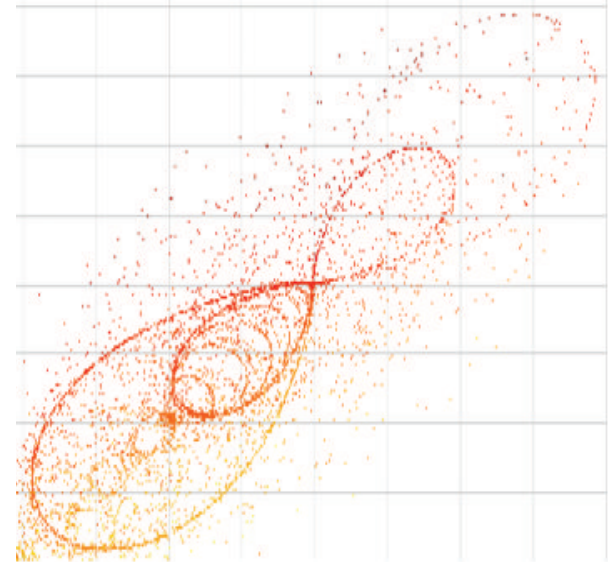

(b)

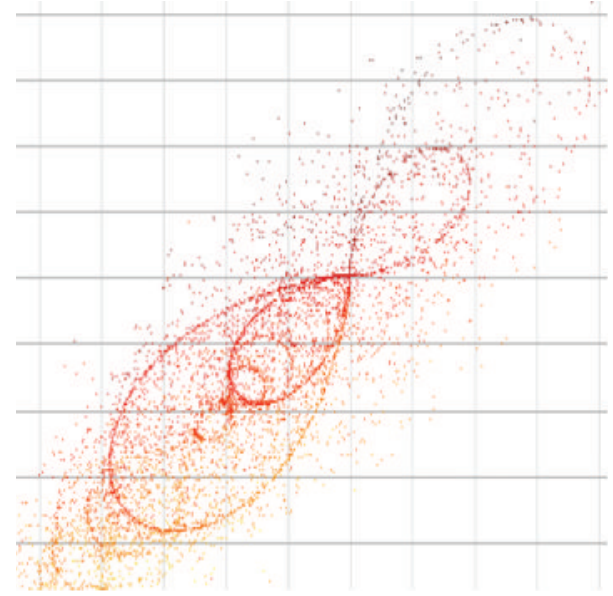

(d)

Figure 8: (a) $R=1.4$, heating rate of $5 \mathrm{~K} / \mathrm{min}$, (b) $R=1.4$, heating rate of $10 \mathrm{~K} / \mathrm{min}$, (c) $R=1.4$, heating rate of $15 \mathrm{~K} / \mathrm{min}$, and (d) $R=1.4$, heating rate of $20 \mathrm{~K} / \mathrm{min}$. (The horizontal axis and vertical axis is, respectively, $x(t)$-DTG and $x(t+1)$-DTG, the colour as dimension).

mutual information has decreased about 75\% from 0 to 1 and about $25 \%$ from 1 to 5 . Comparing the results of Figures 6 (a) and $6(\mathrm{~b})$, it shows that the case for $t=1$ has speeded out the important internal structure; on the contrary, the result of Figure 6(c) shows that a lot of information hidden up for $t=3$. Therefore, the most appropriate for time delay is $t=1$.

\subsection{Results and Discussion of Phase Space Reconstruction.} The DTG phase space reconstruction of Huimin ore with alkalinity ratio of five (heating rate of $10 \mathrm{~K} / \mathrm{min}$ ) was obtained; the calculated results in VRA (Visual Recurrence analysis) software [17] were shown in Figure 7.

It can be seen from Figure 7 that the DTG phase portraits have showed structure with pairs of " $\infty$ " in the smelting reduction process and distributed symmetrically in a square area; alkalinity has little influence on graphics feature.

The condition with better smelting reduction (alkalinity $R=1.4$ ) was selected to experiment under four different heating rates, $5,10,15$, and $20 \mathrm{~K} / \mathrm{min}$, respectively; the results of its DTG phase portrait were shown in Figure 8.
As can be seen from Figure 8, with different heating rates of the same alkalinity, the phase portraits have showed the attractor structural features with the same shape, double " $\infty$ " structure. Its good reproducibility gave expression to the regularity of the DTG phase portrait characteristic. In addition, the internal " $\infty$ " has expressed weak self-similar structure.

As can be seen from Figure 9, the length of the double " $\infty$ " graph has increased with the increase of heating rate, it was provided almost with linear relationship. The maximum width is maintained at about $0.2 \times 2^{3 / 2}$ that indicated the graphics are stretched with the heating rate to speed up and also has verified the intensity of phase transition [10].

2.5. Recurrence Plot Analysis. The recurrence plot proposed by Belaire-Franch and Contreras [18] is mainly used to describe predictability of the time series and to describe its periodic characteristics with visual image. In general, the future state in periodicity system is completely predictable; chaotic dynamical system is of short-term predictability; 


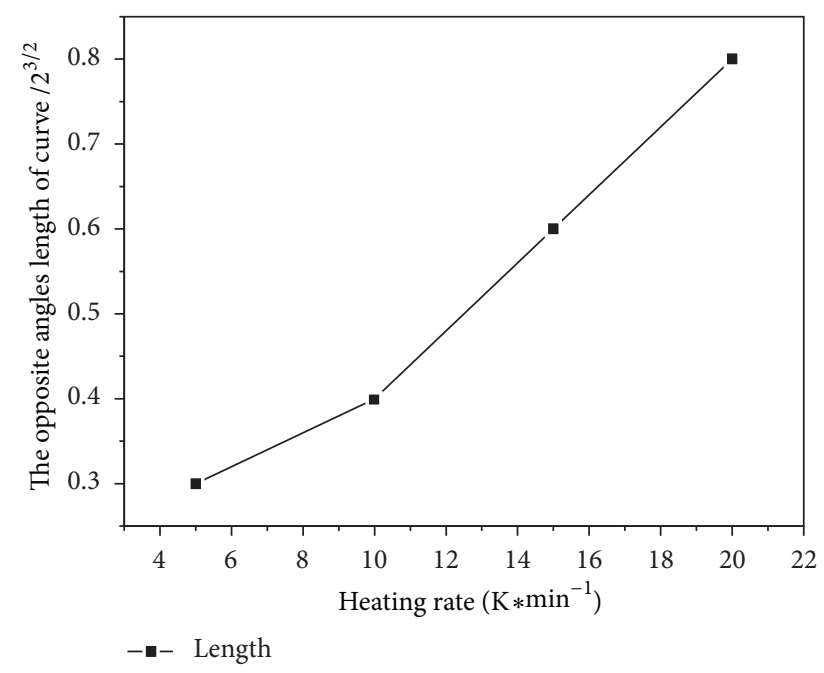

FIGURE 9: The relation of opposite angles length of graph and heating rate.

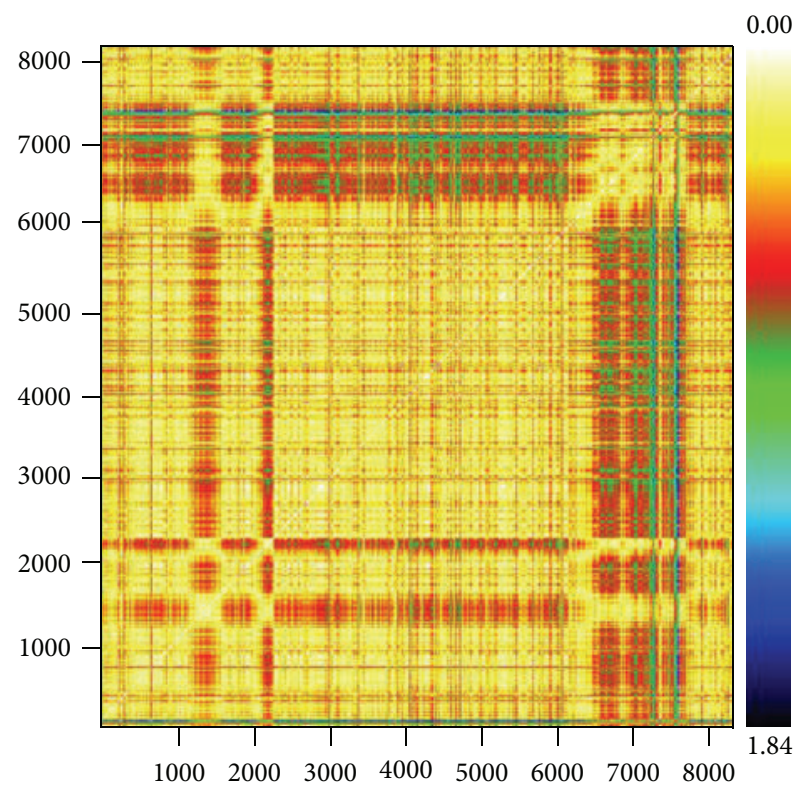

FIgURE 10: The recurrence plot of the DTG.

the stochastic system is completely unpredictable. Thus, the recurrence plot can distinguish the periodic system, chaotic dynamical system, and stochastic system.

The recurrence plot was depicted with reconstructed phase space by the delay time $t=1$ and embedding dimension $m=2$. Horizontal and vertical coordinates in the figure expressed the phase point, when the distance between two points corresponding to horizontal, the vertical axis is less than a relatively small number $\varepsilon_{i}$ given, marked with the $i$ th color corresponding to the location. Theory and experiments had showed that the recurrence plot of the random time series is irregularly. Therefore, it can be seen from Figure 10 that the DTG time series showed randomness or complexity in the smelting reduction process, the sequence tended to be chaotic time series, that is, DTG time series

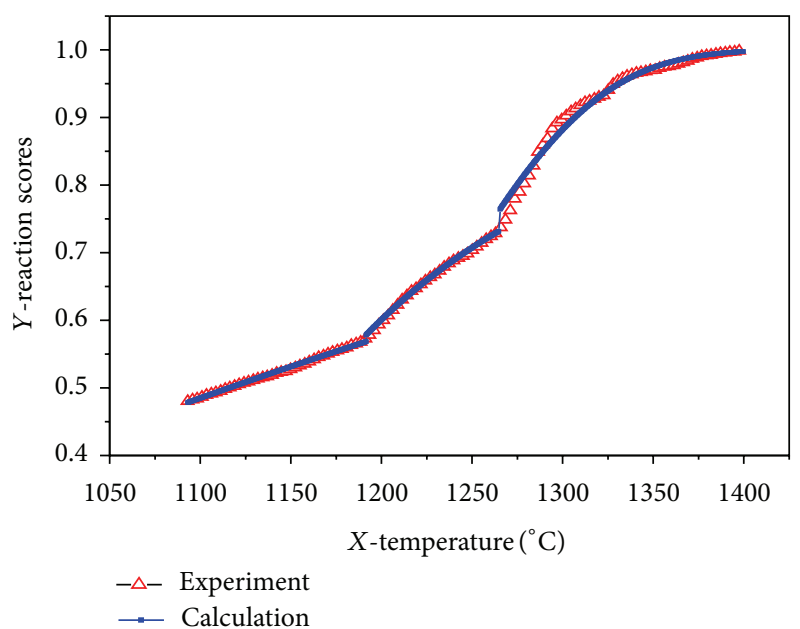

FIGURE 11: Comparison between the measured and model calculated results on reaction fraction of the reduction phase (prereduction and final reduction).

had the nonlinear dynamic random and chaotic, complex characteristics.

\section{Mathematical Model and Analysis of Dynamic System}

The phase portrait of the smelting reduction process reconstructed by the experimental data DTG has the statistical characteristics of the attractor that is double " $\infty$ " structured and nested inside and outside. To reveal better the underlying law of smelting reduction process and its physical and chemical significance, the authors would attempt to build a mathematical equation of the attractor.

3.1. Mathematical Model. Considering that higher degree agreement of the most probable mechanism functions with the experimental data [19], which was from thermal analysis in the various stages of reaction, its function models were employed to establish the structural equation of the attractor, so that to explore the equations of mathematical physics of the graphic structure. The measured and calculated results of various stages were shown in Figure 11.

It can be seen from the Figure 11 that the model calculated and measured values had matched well and the kinetic model equation could express the reaction process of the smelting reduction process. Therefore, the DTG expressions were used to explore the mathematic equation of the attractor with the above-mentioned model.

Taken prereduction stage as an example, the reaction mechanism function $g(\alpha)=[-\ln (1-\alpha)]^{4}$ was obtained. The relationship of the reaction fraction changed with temperature was shown in (5) [19]. Consider

$$
x=1-e^{-\left[(A R / \beta E)(1-(2 R T / E)) T^{2} e^{-E / R T}\right]^{1 / 4}} .
$$

After substituting (5) with $A=a_{1} \beta /\left(a_{2}-T\right)$ [19] and $a=R / E$, where $g(\alpha)$ is the integral mechanism function; $A$ 


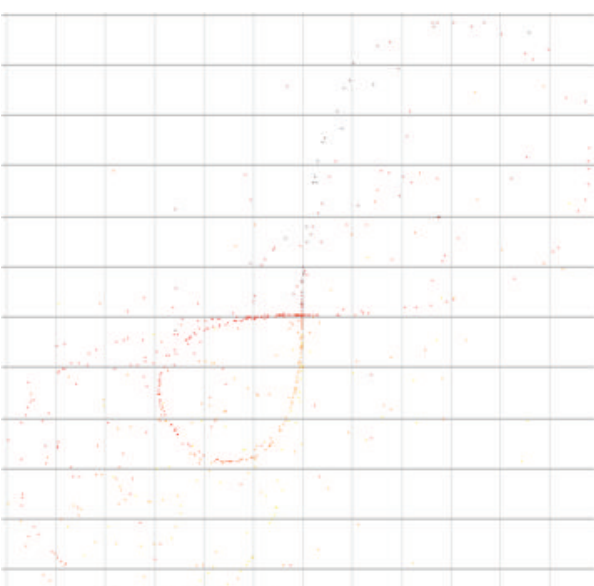

(a)

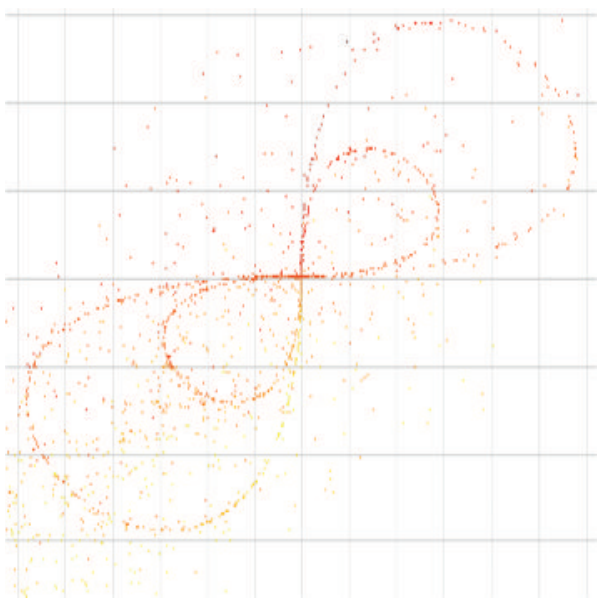

(c)

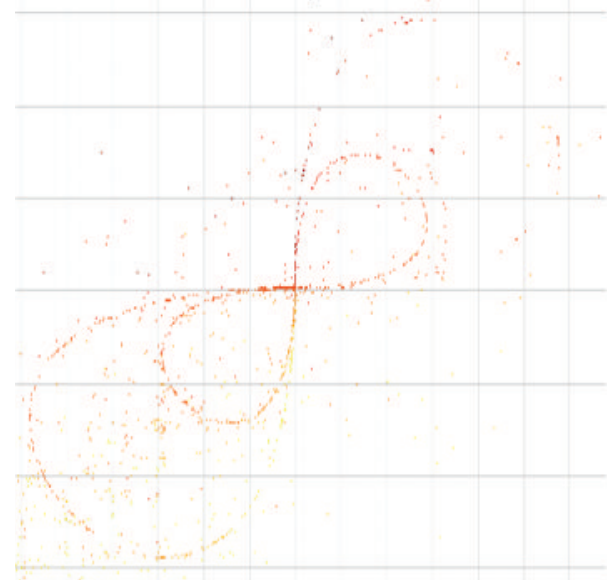

(b)

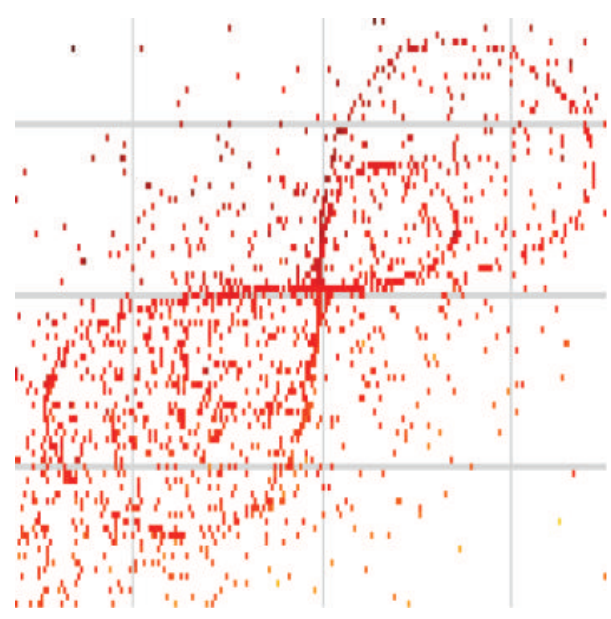

(d)

FIGURE 12: (a) The initial low temperature segment, (b) the first intermediate process, (c) the second intermediate process, and (d) the third intermediate process. (The horizontal axis and vertical axis are, respectively, $x(t)$-DTG and $x(t+1)$-DTG, the colour indicated the dimension).

implies the preexponential factor; $E$ is the apparent activation energy, $R$ represents the gas constant 8.314; $T$ is the heating temperature; and $\beta$ is the heating rate. So (6) is got by finishing. Consider

$$
x=1-e^{-\left[\left(a_{1} a /\left(a_{2}-T\right)\right)(1-2 a T) T^{2} e^{-1 / a T}\right]^{1 / 4}} .
$$

The derivative of (6) to $T$ is expressed as (7)

$$
\begin{aligned}
f(T)=\frac{d x}{d T}=e^{-\left[\left(a_{1} a /\left(a_{2}-T\right)\right)\left(T^{2}-2 a T^{3}\right) e^{-1 / a T}\right]^{1 / 4}} \\
\times \frac{1}{4}\left[\frac{a_{1} a}{a_{2}-T}\left(T^{2}-2 a T^{3}\right) e^{-1 / a T}\right]^{-3 / 4} \\
\times\left[\frac{a_{1} a\left(2 a_{2} T-T^{2}+6 a_{2} a T^{2}+4 a T^{3}\right)}{\left(a_{2}-T\right)^{2}}\right. \\
\left.+\frac{a_{1}(1-2 a T)}{a_{2}-T}\right] e^{-1 / a T} .
\end{aligned}
$$

Thus, $f(T)$ would express the apparent reaction rate model.
Therefore, the above-mentioned method though reasonable could not correctly express DTG data. The main reason is the complexity and dynamic randomness of the reaction in the smelting reduction process of high-phosphorus iron ore. Thus, dynamic system method by time series has an advantage of characterizing dynamic behavior.

3.2. Characterization and Analysis of Dynamic System. The diversity of ore raw material composition and complexity of the chemical reaction combined with the recurrence plot analysis have showed that the system is stochastic dynamical systems; in the meanwhile it is the discrete dynamical system determined by characteristics of the attractor and discrete point, So it can be concluded that it belongs to the random discrete dynamic system [20-23]. The statistical regularities of the dynamical system for each stage were preliminary analyzed to obtain kinetic characteristic. The analytical results of the various stages were shown in Figure 12.

It can be inferred from Figure 12(a), the points inside " $\infty$ " within the curve of double " $\infty$ " characterized that the smelting reduction system came into the stage of interfacial 
chemical reaction control, the reduction process of iron ore generally came into the stage of chemical reaction control at low temperature phase [24]; it can be inferred from Figures 12(b), 12(c), and 12(d) that points outside " $\infty$ " characterized the system came into the stage of diffusioncontrolled [25], while the points scattered around the curve characterized the stage of the mixed control. In addition, due to the diversity of ore ingredients and the complexity of the chemical reaction, dynamic characteristics of the dynamical system restored by the DTG coupled and integrated with the results of the various reaction kinetics control (they are shown in Figures 8(b), 8(c), and 8(d)), the points distributed around the curve were the transitional situation of mixed control.

\section{Conclusion}

TGA experiment on smelting reduction of the Huimin highphosphorus iron ore was made with five different alkalinities and four kinds of different heating rates at the same alkalinity, the derivative thermogravimetric (DTG) data have been obtained from the experiments. Then the phase space reconstruction analysis is found as follows.

(1) The DTG phase portrait by first-order delay has clear graphical structural feature of double " $\infty$ " attractor. It is very profound that statistical properties with inside and outside " $\infty$ " of the attractor had verified two kinetics cases of chemical reaction control stage and diffusion-controlled.

(2) The recurrence plot of the DTG time series showed nonlinear dynamic random and chaotic, complex characteristics.

(3) There is a significant error when DTG equation was deduced by the kinetic mechanism functions of thermal analysis. It indicated that a simple mechanism functions could not express complex reaction in the iron smelting reduction process. The DTG data is certainly a true reflection of the details of the smelting process, but it is coupling and integration of various reactions; the dynamic control of the situation corresponding to the process is comprehensive control of the situation with all kinds of elementary reactions. To this end, it is the correct way to explore the various stages of the statistical law by the ergodicity of the system, which reflects the superiority of the phase space reconstruction method.

The equations of mathematical physics for the attractor has not established accurately, every exaction cannot be reflect in the attractor, they are the faultiness of our method and we will develop future work combined these two aspects.

\section{Conflict of Interests}

The authors declare that there is no conflict of interests regarding the publication of this paper.

\section{Acknowledgment}

This work This work was supported by the Startup Foundation for Doctors (no. PXY-BSQD-2014001) and The Youth
Foundation of Ping Ding Shan University (No.PXY-QNJJ2014008) to which the authors are greatly obliged.

\section{References}

[1] H.-P. Wiendahl and J. Worbs, "Simulation based analysis of complex production systems with methods of non-linear dynamics," Journal of Materials Processing Technology, vol. 139, no. 1-3, pp. 28-34, 2003.

[2] S. Yagiz and C. Gokceoglu, "Application of fuzzy inference system and nonlinear regression models for predicting rock brittleness," Expert Systems with Applications, vol. 37, no. 3, pp. 2265-2272, 2010.

[3] Z. Deng, L. Yang, J. Yu, and G. Luo, "An inverse problem of identifying the coefficient in a nonlinear parabolic equation," Nonlinear Analysis, Theory, Methods and Applications, vol. 71, no. 12, pp. 6212-6221, 2009.

[4] J. Shi, E. Donskoi, D. L. S. McElwain, and L. J. Wibberley, "Modelling the reduction of an iron ore-coal composite pellet with conduction and convection in an axisymmetric temperature field," Mathematical and Computer Modelling, vol. 42, no. 1-2, pp. 45-60, 2005.

[5] D. Tao, "The kinetic models of chemical reaction of fluids on rough surfaces," Acta Metallurgica Sinica, vol. 37, no. 10, pp. 1073-1078, 2001.

[6] D. P. Tao, "Fractal pore diffusion model of fluids in porous media," Acta Metallurgica Sinica, vol. 13, no. 3, pp. 877-883, 2000.

[7] D. Huang, X. Yang, T. Yang, and L. Kong, "Kinetics and mathematical model for reduction process of iron ore briquette containing carbon," Acta Metallurgica Sinica, vol. 32, no. 6, pp. 629-636, 1996.

[8] D. B. Huang and L. T. Kong, "Kinetic model for firing hematite pellet containing solid fuel," Iron and Steelmaking, vol. 30, no. 4, pp. 1-6, 1995.

[9] Q. Wang, Z. Yang, J. Tian, W. Li, and J. Sun, "Mechanisms of reduction in iron ore-coal composite pellet," Ironmaking and Steelmaking, vol. 24, no. 6, pp. 457-460, 1997.

[10] M. Cross, T. N. Croft, G. Djambazov, and K. Pericleous, "Computational modelling of bubbles, droplets and particles in metals reduction and refining," Applied Mathematical Modelling, vol. 30, no. 11, pp. 1445-1458, 2006.

[11] P. Mark, M. Cross, and P. Schwarz, "A two-dimensional steadystate simulation model for a lead blast furnace," in Proceedings of the 3rd International Conference on CFD in the Minerals and Process Industries, pp. 641-644, CSTRO, Melbourne, Australia, December 2003.

[12] X. Ma, M. Jiang, and D. Wang, "Kinetics and model of reaction process of iron ore-coal pellet," Journal of Northeastern University, vol. 23, no. 5, pp. 440-443, 2002.

[13] A. M. Fraser and H. L. Swinney, "Independent coordinates for strange attractors from mutual information," Physical Review A, vol. 33, no. 2, pp. 1134-1140, 1986.

[14] J. W. Dippner, R. Heerkloss, and J. P. Zbilut, "Recurrence quantification analysis as a tool for characterization of nonlinear mesocosm dynamics," Marine Ecology Progress Series, vol. 242, pp. 29-37, 2002.

[15] D. Chen, Y. Liu, and X. Ma, "Parameter joint estimation of phase space reconstruction in chaotic time series based on radial basis function neural networks," Acta Physica Sinica, vol. 61, no. 10, Article ID 100501, 2012. 
[16] D. Chen and W. Han, "Prediction of multivariate chaotic time series via radial basis function neural network," Complexity, vol. 18, no. 4, pp. 55-66, 2013.

[17] C. L. Webber Jr., "Recurrence quantification analysis of nonlinear dynamical systems," COMMENT, vol. 1, pp. 1-94, 2005.

[18] J. Belaire-Franch and D. Contreras, "Recurrence plots in nonlinear time series analysis: free software," Journal of Statistical Software, vol. 7, pp. 1-18, 2002.

[19] G. F. Fan, S. Qing, H. Wang, and W.-C. Hong, "The kinetic model of direct melting reduction process of huimin iron ore," Mineral Processing and Extractive Metallurgy, vol. 122, no. 2, pp. $1-8,2013$.

[20] T. Trzaskalik and S. Sitarz, "Discrete dynamic programming with outcomes in random variable structures," European Journal of Operational Research, vol. 177, no. 3, pp. 1535-1548, 2007.

[21] T. J. J. van den Boom, B. Heidergott, and B. de Schutter, "Complexity reduction in MPC for stochastic max-plus-linear discrete event systems by variability expansion," Automatica, vol. 43, no. 6, pp. 1058-1063, 2007.

[22] R. Iwankiewicz, "Equations for probability density of response of dynamic systems to a class of non-Poisson random impulse process excitations," Probabilistic Engineering Mechanics, vol. 23, no. 2-3, pp. 198-207, 2008.

[23] V. Kontorovich and V. Lyandres, "Dynamic systems with random structure: an approach to the generation of nonstationary stochastic processes," Journal of the Franklin Institute, vol. 336, no. 6, pp. 939-954, 1999.

[24] E. R. Hudson, C. Ticknor, B. C. Sawyer et al., "Production of cold formaldehyde molecules for study and control of chemical reaction dynamics with hydroxyl radicals," Physical Review A, vol. 73, no. 6, Article ID 063404, 2006.

[25] D. C. Kundaliya, S. B. Ogale, S. E. Lofland et al., "On the origin of high-temperature ferromagnetism in the low-temperatureprocessed Mn-Zn-O system," Nature Materials, vol. 3, no. 10, pp. 709-714, 2004. 


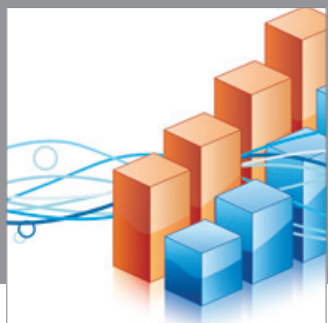

Advances in

Operations Research

mansans

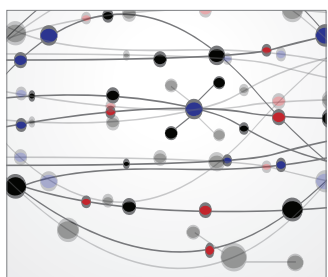

The Scientific World Journal
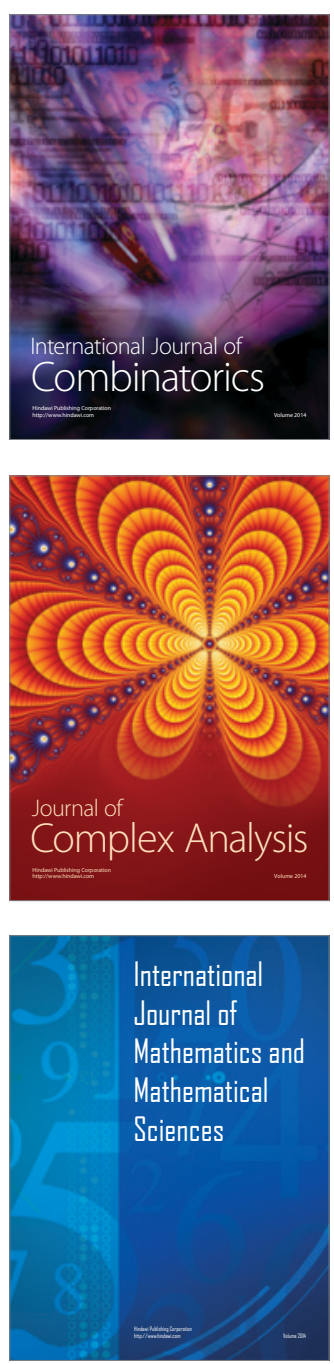
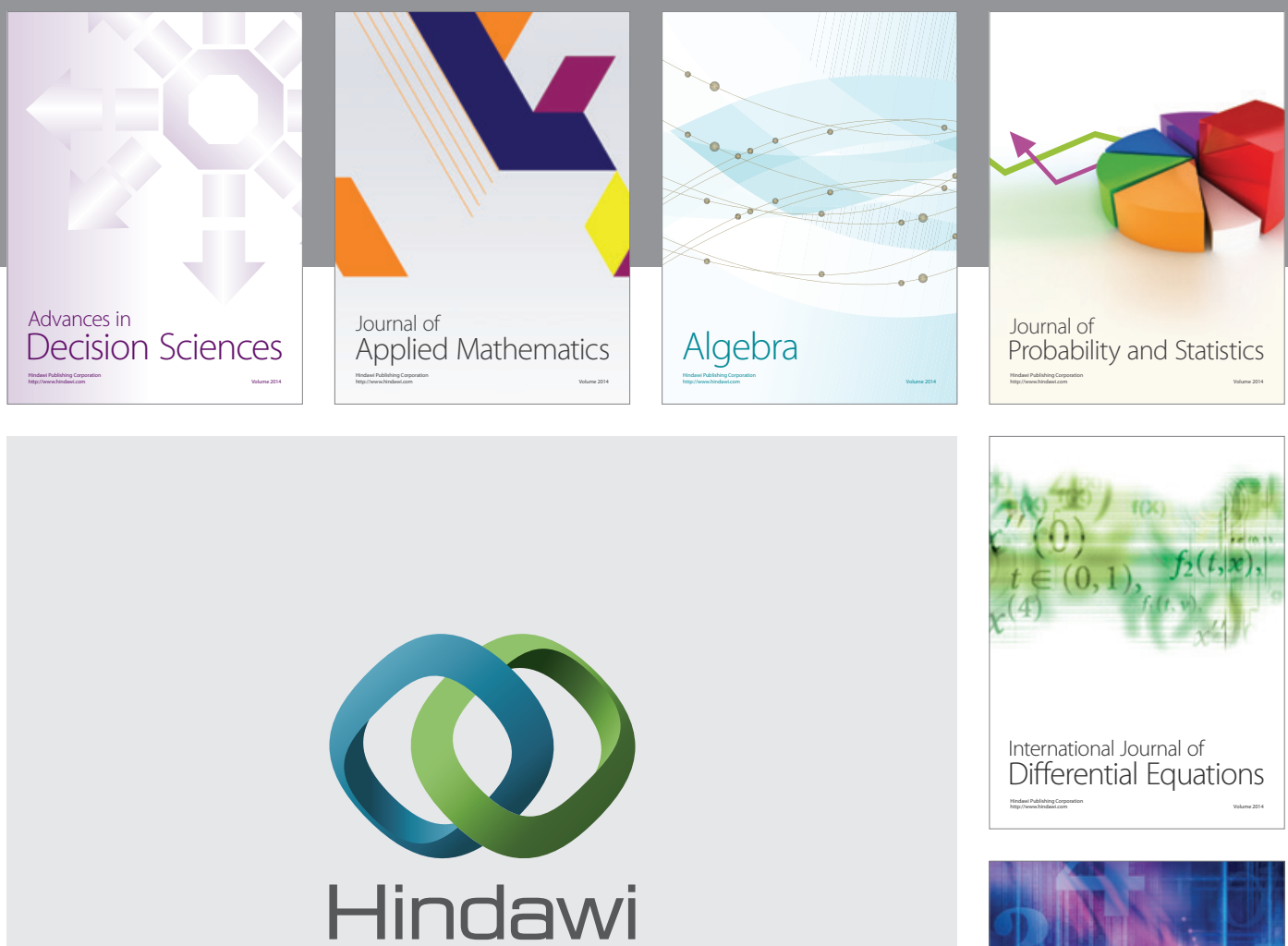

Submit your manuscripts at http://www.hindawi.com
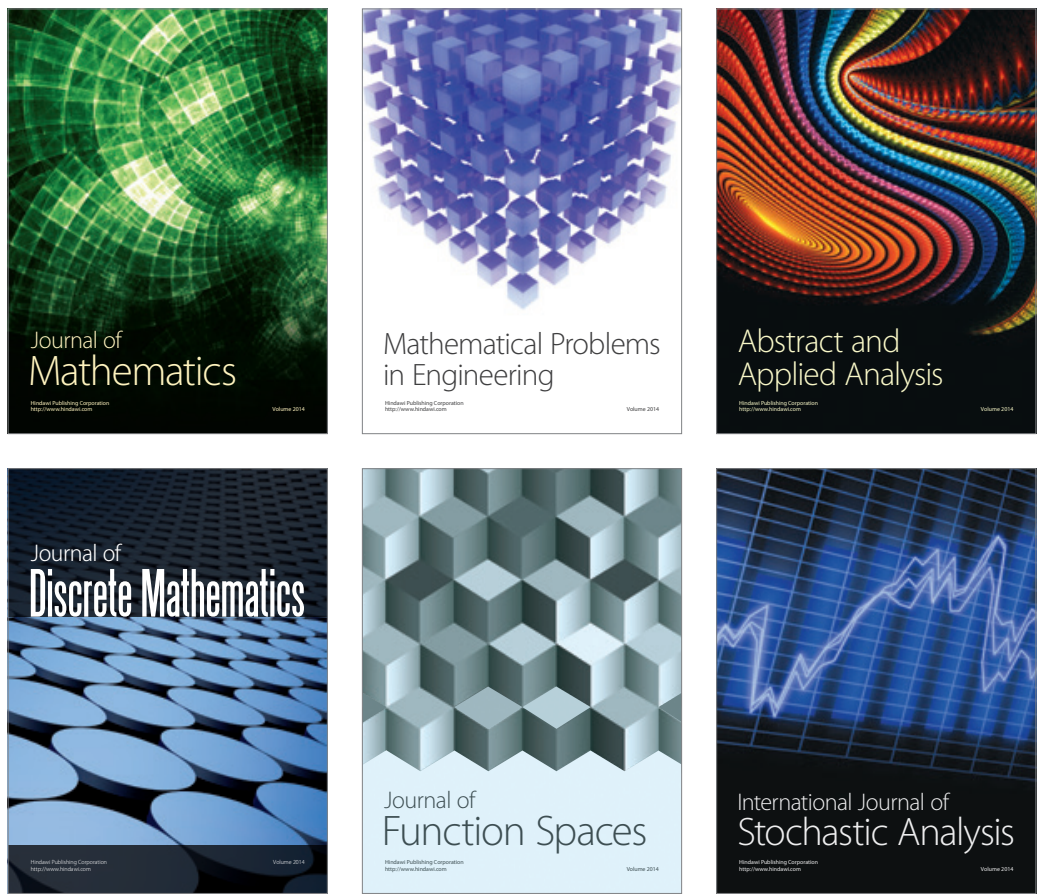

Journal of

Function Spaces

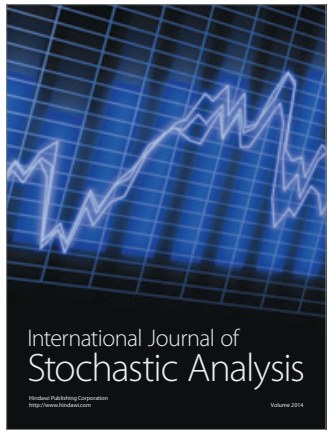

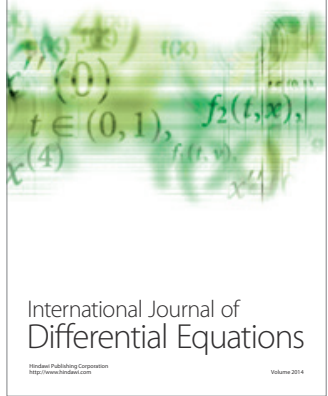
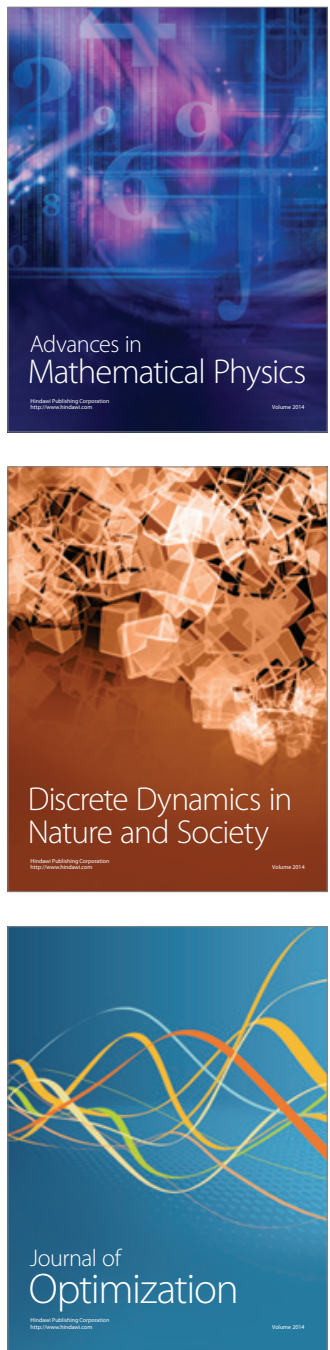